\title{
RASTREIO DA CONDIÇÃO NEUROPSICOLÓGICA DE PROFISSIONAIS DE SAÚDE NA LINHA DE FRENTE AO COMBATE DO COVID-19: DADOS PRELIMINARES
}

Screening the neuropsychological condition of health professionals on the frontlines of the

COVID-19 fight: Preliminary data

Tamires N. Teixeira ${ }^{1}$

Zenóbio Cosme Gonçalves Ferreira ${ }^{2}$

Petra R. Paul

Roberto $\mathrm{Cruz}^{3}$

Rosane Porto Seleme ${ }^{4}$

Rachel Schlindwein-Zanini ${ }^{5}$

\section{Resumo}

Introdução: uma pandemia pode promover manifestações cognitivas e comportamentais, afetando especialmente profissionais de saúde no enfrentamento do COVID-19. Objetivo: investigar a condição neuropsicológica de profissionais de saúde na linha de frente ao combate do COVID-19 no sul do estado de Santa Catarina, durante o pico da pandemia, prévio ao período vacinal. Metodologia: aplicação de versão piloto online do NEUROPSIC-R (considerando escore mínimo de 43 pontos para a identificação/suspeita de alterações neuropsicológicas (valores mínimos de 19 e máximo de 76 pontos), respondido voluntariamente, assim como as perguntas acerca de fadiga e contágio por COVID-19. Resultados: 62 profissionais (média 35,7 e desvio padrão de 7,5 anos), ambos os sexos. A maioria eram Enfermeiros (38,7\%), seguido de Agentes comunitários, Técnicos, de Médicos, Dentistas e Psicólogos, higienizadores, entre outros (8\%). No grande grupo, observou-se escores deficitários no NEUROPSIC-R, especialmente em oscilação do humor e depressão (66\% dos sujeitos), dificuldade de concentração (63\%), de atenção $(56,5 \%)$ e em memória recente $(55 \%)$. Adicionalmente, questiona-se acerca da preocupação com o avanço da pandemia e sobre a contaminação de familiares pela COVID-19, tendo $100 \%$ de respostas afirmativas em ambas. Foi questionado, também, sobre fadiga e cansaço, com afirmação em $84 \%$ dos respondentes, tendo uso de psicotrópicos para ansiedade (com 34\% de afirmações). Conclusão: evidenciou-se relevante frequência de oscilação do humor, sintomas depressivos e ansiedade, dificuldade de concentração e fadiga, bem como alta preocupação com contágio nestes

\footnotetext{
1 Psicóloga aluna do Mestrado Profissional em Saúde Mental e Assistência Psicossocial - CCS/UFSC. E-mail: taminascimentoteixeira@gmail.com

2 Graduando em Psicologia pela Universidade Federal de Santa Catarina, membro do Núcleo de Neuropsicologia e Saúde - HU/UFSC, Florianópolis, SC, Brasil. E-mail: zenobiopsi@gmail.com

${ }^{3}$ Psicólogo psicometrista doutor em Eng.de Produção - UFSC, Professor do Depto e do Programa de Pós-Graduação em Psicologia da UFSC. Líder do Laboratório Fator Humano (UFSC) e pesquisador do Núcleo de Pesquisa em Neuropsicologia e Saúde - HU/UFSC. E-mail: robertocruzdr@gmail.com

${ }^{4}$ Médica neuroanatomista mestre em Neurociências e doutora em Eng. de Produção - UFSC. Professora do Depto de Ciências Morfológicas (CCB/UFSC). Membro do Núcleo de Neuropsicologia e Saúde - HU/UFSC. E-mail: rosane.seleme@ufsc.br

${ }^{5}$ Neuropsicóloga do Hospital Universitário da UFSC, preceptora de residência (RIMS) e Professora permanente do Programa de Mestrado Profissional em Saúde Mental e Assistência Psicossocial CCS/UFSC, Coordenadora do Núcleo de Neuropsicologia e Saúde - HU/UFSC. Professora da Faculdade de Medicina - UNISUL. Doutora em Ciências da Saúde/Medicina (área Neurociências) - PUCRS. E-mail: rachelsz@floripa.com.br
} 
profissionais, repercutindo na sua condição cognitiva e emocional. Também se constatou a boa aplicabilidade do NEUROPSIC-R, inclusive na versão online.

Palavras-chave: Neuropsicologia; COVID-19; Profissionais de saúde.

\section{Abstract}

Introduction: a pandemic can promote cognitive and behavioral manifestations, especially affecting health professionals in coping with COVID-19. Purpose: to investigate the neuropsychological condition of health professionals on the front line to combat COVID-19 in the southern state of Santa Catarina, during the peak of the pandemic, prior to the vaccination period. Methodology: application of an online pilot version of the NEUROPSIC-R (considering a minimum score of 43 points for the identification/suspicion of neuropsychological disorders (minimum values of 19 and maximum of 76 points), answered voluntarily, and questions about fatigue and contagion by COVID-19. Results: 62 professionals (mean 35.7 and standard deviation of 7.5 years), both genders. Most were Nurses $(38.7 \%)$, followed by Community Workers, Technicians, Physicians, Dentists and Psychologists, sanitizers, among others (8\%). In the all group, there were deficient scores on the NEUROPSIC-R, especially in mood swings and depression (66\% of subjects), difficulty in concentration (63\%), in attention (56.5\%) and in recent memory $(55 \%)$. Additionally, he was asked about the concern with the progress of the pandemic and about the contamination of family members by COVID-19, with $100 \%$ of affirmative answers in both. Also, about fatigue and tiredness, with affirmation in $84 \%$ of respondents, using psychotropic drugs for anxiety (with $34 \%$ of the group). Conclusion: there was a relevant frequency of mood swings, depressive symptoms and anxiety, difficulty concentrating and fatigue, as well as a high concern with contagion in these professionals, affecting their cognitive and emotional condition. The good applicability of NEUROPSIC-R was also verified, including in the online version.

Keywords: Neuropsychology; COVID-19; Healthcare professionals.

\section{Introdução}

O contexto pandêmico é marcado por incertezas e inseguranças, causando temor em boa parcela da população desde a declaração de Emergência de Saúde Pública da Organização Mundial de Saúde no início de 2020, com importância internacional, sendo este o mais alto nível de alerta. No centro dessa conjuntura encontram-se os trabalhadores da saúde, contemplando variados profissionais. No Brasil foi possível observar evidências do alto risco de contágio a que estes trabalhadores estão expostos. A partir do estudo de Barroso et al. (2020), foi realizado um mapeamento desse risco e constatado que esse grupo apresentou um perigo de contágio de $97 \%$ a $100 \%$ durante suas atividades profissionais. É importante salientar que o presente estudo analisou diversas inserções profissionais, desde técnicos de saúde bucal até médicos.

A partir disso, são diversas as possíveis consequências envolvendo adoecimento psíquico e alterações neuropsicológicas. Se por um lado esses profissionais estão 
expostos à exaustão física e mental, por outro eles são peça chave para dirimir os impactos da pandemia. Os prejuízos à saúde mental que estes trabalhadores vivenciam são potencializados por diversos fatores, que vão desde sofrimento envolvendo a perda de colegas, de pacientes, receio da própria contaminação ou de transmissão a pessoas próximas e familiares até a própria dificuldade de tomada de decisão em relação a atividades laborais.

Considerando que os profissionais de saúde envolvidos no enfrentamento da pandemia, estão expostos cotidianamente ao risco de adoecer pelo coronavírus, ao cansaço físico e estresse psicológico e insuficiência e/ou negligência com relação às medidas de proteção (TEIXEIRA et al, 2020), e que o cenário de uma epidemia/pandemia pode promover manifestações depressivas, ansiogênicas, psicóticas, de luto, de piora cognitiva, de solidão e impotência (SCHLINDWEIN-ZANINI; PAUL, 2020), faz-se importante, então, direcionar esforços para rastreio e identificação de quais repercussões estão sendo experimentadas por estas pessoas para, a partir disso, oferecer a melhor atenção à saúde desses profissionais, que são fundamentais para a saúde de todos.

\section{Objetivo}

Investigar a percepção da própria condição neuropsicológica de profissionais de saúde na linha de frente ao combate do COVID-19 no sul do estado de Santa Catarina, durante o pico da pandemia, prévio ao período vacinal.

\section{Metodologia}

A coleta de dados ocorreu através de aplicação de versão piloto online do NEUROPSIC-R (considerando escore mínimo de 43 pontos para a identificação/suspeita de alterações neuropsicológicas (valores mínimos de 19 e máximo de 76 pontos) respondido voluntariamente, assim como as perguntas acerca de fadiga e contágio por COVID-19.

\section{Resultados}

O número de profissionais da saúde que participaram foi de 62 (média 35,7 e desvio padrão de 7,5 anos), ambos os sexos (84\%, 52 mulheres), 16\%, 10 homens). A maioria eram Enfermeiros (38,7\%), seguido de Agentes comunitários (19,4\%), Técnicos (em Enfermagem ou Téc. Saúde Bucal) (16,1\%), de Médicos, Dentistas e Psicólogos 
(cada com 4,8\%), além de higienizadores (3,2\%) entre outros (8\%). Destes, 42,2\% são casados. No grande grupo, observou-se escores deficitários no NEUROPSIC-R, especialmente em oscilação do humor e depressão (66\% dos sujeitos), dificuldade de concentração (63\%), de atenção (56,5\%) e em memória recente (55 \%). No entanto, os domínios mais preservados foram de autonomia (84\%), orientação espacial $(88,7 \%)$ e atividades manuais (82,3\%). Adicionalmente, duas perguntas foram realizadas sobre COVID-19 ("Você se sente preocupado(a) com o avanço da pandemia COVID-19?" e "Você se sente preocupado(a) com a contaminação de familiares pela COVID-19?"), tendo $100 \%$ de respostas afirmativas em ambas. Além de uma pergunta sobre fadiga e cansaço, com afirmação em $84 \%$ dos respondentes e uso de psicotrópicos para ansiedade (com $34 \%$ de afirmações).

\section{Discussão}

Ao observar os dados do presente estudo, identificou-se que, Teixeira et al. (2020) referiram a frequência do transtorno de ansiedade generalizada, estresse crônico, sentimento de impotência, exaustão ou esgotamento dos trabalhadores frente à intensa carga de trabalho, tendendo a piorar num contexto de carência de mão-de-obra na eventualidade do contágio de COVID-19. Os achados da presente pesquisa corroboram, também, com o relato recorrente do aumento dos sintomas de ansiedade, depressão, perda da qualidade do sono, aumento do uso de drogas, sintomas psicossomáticos e medo destes se infectarem ou transmitirem aos membros da família (FIOCRUZ, 2020).

Inclusive, Almondes, Agudelo e Jiménez-Correa (2021) explicam que estes profissionais de saúde podem experimentar a dessincronização de seu ritmo circadiano e um desequilíbrio entre o esforço e a recompensa das horas de trabalho, resultando em impacto na capacidade de trabalho, agravado pela falta de equipamentos de proteção individual (EPIs), poucos recursos e infraestrutura precária e medo de contrair o vírus e contaminar familiares. Algumas consequências são privação de sono, insônia crônica, distúrbios do sono relacionados ao estresse e distúrbio de estresse pós-traumático (PSTD). Essas alterações do sono afetam criticamente a saúde mental, precipitando ou perpetuando ansiedade, estresse e depressão, resultando na incapacidade de regular as emoções positivas e negativas. Os distúrbios do sono pré-existentes são um importante fator de risco para o desenvolvimento e manutenção de PSTD quando os indivíduos são expostos a um importante estressor, como uma pandemia de COVID-19. Ao mesmo 
tempo, a forma como um indivíduo regula a emoção associada às preocupações durante o funcionamento diurno impacta o sono noturno, precipitando e perpetuando dificuldades para dormir. Todas essas mudanças no sono e na regulação emocional também alteram o sistema imunológico. A privação do sono está comumente associada a doenças inflamatórias crônicas, devido às dessincronizações nos ritmos circadianos, causando possíveis distúrbios psicofisiológicos e comprometimento da homeostase neuroimune-endócrina.

De forma relevante, corroborando com tais dados, foram aqui observadas uma elevada frequência de oscilação do humor, sintomas depressivos e ansiedade, dificuldade de concentração e fadiga, bem como alta preocupação com contágio nestes profissionais, repercutindo na sua condição cognitiva e emocional, carentes de medidas de saúde mental efetivas. Destaca-se que foi constatada a boa aplicabilidade do NEUROPSIC-R, inclusive da versão online, para rastreio desses construtos. O estudo de Davey et al (2020) também utilizou um questionário destinado a profissionais de saúde em atuação presencial, que relataram vários sintomas de prejuízos no desempenho cognitivo e físico, agravados pelo estresse e calor (do ambiente e do uso de EPIs).

É preciso lembrar que os sistemas de saúde, tanto a nível nacional quanto mundial, vem se adaptando a partir de um cenário de sobrecarregamento de trabalho.

Tal panorama constatado nesse estudo corrobora achados de Hummel et al. (2021), quando salientam que a COVID-19 representa um grande desafio para a saúde mental dos profissionais que trabalham, uma vez que uma proporção considerável destes apresentaram altos valores para depressão, ansiedade e estresse, devendo-se oferecer ajuda suficiente a todos os grupos ocupacionais, com ênfase em estratégias eficazes de enfrentamento. Em outros estudos, como o realizado por Pereira et al. (2020), foram encontradas alterações psicológicas semelhantes, como humor deprimido e a sensação de alto risco de contaminação dos trabalhadores da saúde. Em um levantamento transversal realizado por Huang e Zhao (2020) destinado a analisar os impactos na saúde devido à COVID-19 entre diversos setores da sociedade, foi observado que os profissionais de saúde tiveram maior prejuízo na qualidade do sono, inclusive quando esses dados eram confrontados com os de outros grupos ocupacionais.

No cerne dessa questão, estão os verdadeiros responsáveis pelo funcionamento do sistema de saúde: os profissionais da saúde. A continuação desses serviços só foi possível, pois estes estão enfrentando uma grande demanda de trabalho, além de seus 
próprios receios. Naturalmente, essa conjuntura oferece risco à saúde, tanto mental quanto física, como foram observadas nesse estudo em que foram demonstradas alterações específicas nessas dimensões. A partir disso, podem ser delineadas medidas adequadas para minimizar os impactos na saúde desse grupo de trabalhadores.

\section{Conclusão}

É pertinente salientar o caráter incomum da presente pesquisa, destinada a profissionais da linha de frente em saúde no sul do País, em momento incerto acerca de dados sobre a enfermidade, prognóstico e vacinas. Pesquisa esta que envolveu resiliência na busca de acesso aos respondentes no próprio local de coleta, além de aspectos burocráticos em período pandêmico.

Destaca-se a identificação de distúrbios cognitivos, comportamentais e biológicos, além do comprometimento da qualidade de vida, repercutindo no desempenho laboral e no núcleo familiar dos profissionais de saúde, atuantes presencialmente na pandemia por COVID-19.

Assim, como potencialidade da presente pesquisa, cita-se a produção de conhecimento no mapeamento deste cenário no sul do Brasil, caminho para a construção de uma atenção apropriada e pormenorizada aos trabalhadores da saúde, cada vez mais necessária.

\section{Referências}

ALMONDES, K. M. de; AGUDELO, H. A. M.; CORREA, U. J. Impact of Sleep Deprivation on Emotional Regulation and the Immune System of Healthcare Workers as a Risk Factor for COVID 19: Practical Recommendations From a Task Force of the Latin American Association of Sleep Psychology. Frontiers in Psychology, v. 12, p. 1625, 2021.. doi: 10.3389/fpsyg.2021.564227. PMID: 34093295; PMCID: PMC8172768.

BARROSO B. I. L., et al. Saúde do trabalhador em tempos de COVID-19: reflexões sobre saúde, segurança e terapia ocupacional. João Pessoa: Cadernos Brasileiros de Terapia Ocupacional, v. 28, p. 1093-1102, 2020.

Davey SL, Lee BJ, Robbins T, Randeva H, Thake CD. Heat stress and PPE during COVID-19: impact on healthcare workers' performance, safety and well-being in NHS settings. Journal of Hospital Infection. 2021 Feb;108:185-188. doi:

10.1016/j.jhin.2020.11.027. Epub 2020 Dec 7. PMID: 33301841; PMCID: PMC7720696.

FUNDAÇÃO OSWALDO CRUZ (FIOCRUZ), Brasil. Ministério da Saúde (MS). Saúde mental e atenção psicossocial na pandemia Covid.

Recomendações para gestores 2020. Rio de Janeiro, Brasília: Fiocruz, MS; 2020.
Ciênc. saúde
coletiva
25
(9), Set 2020.
Disponível em: 
http://www.fiocruzbrasilia.fiocruz.br/wpcontent/uploads/2020/04/Sa\%C3\%BAde-Mental. https://doi.org/10.1590/1413-81232020259.19562020

HUANG, Y.; ZHAO, N. Generalized anxiety disorder, depressive symptoms and sleep quality during COVID-19 outbreak in China: a web-based cross-sectional survey. Psychiatry research, v. 288, p. 112954, 2020.

HUMMEL, S. et al. Mental health among medical professionals during the COVID-19 pandemic in eight european countries: Cross-sectional survey study. Journal of medical Internet research, v. 23, n. 1, p. e24983, 2021. doi: 10.2196/24983. PMID: 33411670; PMCID: PMC7817254.

PEREIRA MD, et al. The COVID-19 pandemic, social isolation, consequences on mental health and coping strategies: an integrative review. Revista Research, Society and Development, 2020; 9(5):1-29.

SCHLINDWEIN-ZANINI, R; PAUL, P. R. Cuidados a pessoas com alterações neuropsicológicas nas pandemias. IN: Cruz, R.M. et al. Atenção à saúde mental nas pandemias. 2020. Ed. Ampla.

TEIXEIRA, C. F. de S.et al. A saúde dos profissionais de saúde no enfrentamento da pandemia de Covid-19. Ciência \& Saúde Coletiva, v. 25, p. 3465-3474, 2020. 